\title{
Mês de parição, condição corporal e resposta ao protocolo de inseminação artificial em tempo fixo em vacas de corte primíparas
}

\author{
[Calving date, body condition score, and response to a timed artificial insemination protocol \\ in first-calving beef cows] \\ M. Meneghetti, J.L.M. Vasconcelos \\ Faculdade de Medicina Veterinária e Zootecnia - UNESP \\ Departamento de Produção Animal \\ Caixa Postal 560 \\ 18618-000 - Botucatu, SP
}

\begin{abstract}
RESUMO
No experimento I, foi avaliada a alteração da condição corporal (CC) pré e pós-parto em 155 novilhas inseminadas para parir de setembro a dezembro. A CC foi avaliada mensalmente no pré e pós-parto, de junho a fevereiro. No experimento II, 538 vacas primíparas foram sincronizadas com o protocolo de inseminação artificial em tempo fixo (IATF) que usou estradiol junto ao dispositivo intravaginal de progesterona $\left(\mathrm{CIDR}^{\mathbb{B}}\right)$. As taxas de ciclicidade, sincronização e concepção foram avaliadas por ultra-som. No experimento I, os animais que pariram primeiro tiveram maior $(\mathrm{P}<0,001)$ redução na $\mathrm{CC}$ pós-parto. No experimento II, foi observado maior $\mathrm{CC}(\mathrm{P}<0,0001)$ nos animais com menor número de dias pósparto, maior $(\mathrm{P}<0,05)$ taxa de sincronização nas vacas de melhor $\mathrm{CC}$ e aumento $(\mathrm{P}<0,0001)$ na taxa de concepção proporcional ao aumento na CC (incremento médio na concepção de seis pontos percentuais para cada 0,25 ponto na CC). Não se deve antecipar a parição de novilhas de corte quando se pretende realizar IATF no início da estação de monta subseqüente.
\end{abstract}

Palavras-chave: gado de corte, primípara, condição corporal, inseminação artificial em tempo fixo

\begin{abstract}
In experiment I, it was evaluated the body condition score (BCS) change during pre and post-partum in 155 heifers, inseminated to calve from September to December. The BCS was monthly evaluated from June to February, during the pre and post-partum periods. In experiment II, 538 primiparous cows were synchronized with a timed artificial insemination (TAI) protocol which used estradiol associated with an intravaginal progesterone device $\left(C I D R^{\circledR}\right)$. The cyclicity, synchronization, and conception rates were evaluated by ultrasound. In experiment I, the animals that calved earlier had higher $(P<0.001)$ reduction on BCS. In experiment II, it was observed higher BCS $(P<0.0001)$ in cows with lower days in postpartum, higher $(P<0.05)$ synchronization rate in cows with a better $B C S$, and also an increase $(P<0.0001)$ in conception rate as BCS got better (increase in six percentual points in conception for each increase of 0.25 in BCS). Beef heifers should not calve earlier when is planned to submitt these animals to TAI at the beginning of the next breeding season.
\end{abstract}

Keywords: beef cattle, primiparous, body condition score, timed artificial insemination

\section{INTRODUÇÃO}

Prolongado período de anestro em vacas de corte com cria ao pé é uma das principais causas de perdas econômicas para os pecuaristas, por atrasar a concepção e/ou levar ao descarte por falha reprodutiva. Estudos mostram que a condição nutricional pré e pós-parto influencia a duração do período de anestro (Richards et al., 1986; Ruas et al., 2005).

Recebido em 25 de abril de 2007

Aceito em 24 de março de 2008

E-mail: vasconcelos@fca.unesp.br 
Relatos da década de 70 revelaram que vacas primíparas apresentam período de anestro mais longo (20 a 30 dias) do que vacas multíparas (Wiltbank, 1970; Mossman e Hanly, 1977). Desde então, em fazendas que usam estação de monta (EM), com monta natural ou programas de IA convencional, é prática comum iniciar a EM de novilhas antes do restante do rebanho, a fim de que no ano seguinte, após o primeiro parto, elas disponham de maior período para se recuperar do anestro pós-parto antes do início da estação de monta.

A estratégia de antecipar o parto em vacas primíparas tem como objetivo reduzir a proporção de animais em anestro no início da EM, porém a preocupação com a ciclicidade pode ser minimizada quando se usa inseminação artificial em tempo fixo (IATF) no início da EM. Meneghetti et al. (2005a) não observaram efeito de ciclicidade nas taxas de ovulação e prenhez, sendo de $90,5 \%$ e $51,1 \%$ nas vacas em anestro, respectivamente. Lamb et al. (2001) e Bó et al. (2004) também obtiveram taxas de concepção à IATF acima de $50 \%$ em vacas em anestro.

No Brasil, os partos concentram-se no final do inverno e durante a primavera, ou seja, os animais têm o período final de gestação e início da lactação em épocas de menor disponibilidade de alimento, em termos de quantidade e qualidade, levando à redução das reservas corporais no peri-parto. Esse efeito pode ser mais evidente em vacas de primeira cria, devido a sua maior demanda por nutrientes, por ainda estarem em crescimento e iniciando a primeira lactação (Nutrient ..., 2000).

Estudos mostram o efeito da CC no momento da IATF sobre a taxa de concepção a protocolos hormonais. Cutaia e Bó (2004) verificaram correlação de $90 \%$ entre eles, e animais com melhor CC respondem melhor aos protocolos de IATF, resultando em maiores taxas de concepção (Lamb et al., 2001; Meneghetti et al., 2005b).

A hipótese levantada neste trabalho é a de que vacas primíparas que passam por menor período de restrição alimentar pós-parto, apesar de terem menor número de dias pós-parto (DPP), terão maior CC, melhor resposta ao protocolo de sincronização de ovulação e, conseqüentemente, maior taxa de concepção a IATF no início da estação de monta.
O objetivo deste estudo foi avaliar diferentes momentos de cobrição de novilhas, buscando determinar o momento do melhor desempenho reprodutivo após o primeiro parto, em propriedades que usam IATF no início da estação de monta.

\section{MATERIAL E MÉTODOS}

O experimento I foi delineado para avaliar o efeito do mês de parição na dinâmica da condição corporal de novilhas de corte pré e pósparto em rebanho comercial, localizado no município de Alto Taquari, região sudeste do estado do Mato Grosso. Foram usadas 200 novilhas, sendo 110 da raça Nelore e 90 novilhas $1 / 2$ Nelore $+1 / 2$ Red Angus com, aproximadamente, 24 meses de idade e pesando acima de $300 \mathrm{~kg}$.

Os animais foram divididos aleatoriamente, antes da estação de monta, em dois grupos (I e II) com 100 animais cada, sincronizados e inseminados em duas épocas e depois submetidos à monta natural, visando à distribuição dos partos na estação de parição subseqüente. A IATF do grupo I foi realizada em 29 de novembro de 2003, e as vacas foram submetidas à monta natural de 30 de novembro de 2003 até 28 de fevereiro de 2004; a IATF do grupo II foi realizada em 24 de janeiro de 2004, e as vacas submetidas à monta natural de 25 de janeiro até 28 de fevereiro de 2004. Após o diagnóstico de gestação, realizado em junho de 2004, foram mantidos no experimento 155 animais que vieram a parir entre setembro e dezembro de 2004, sendo 87 animais da raça Nelore e 68 animais $1 / 2$ Nelore $+1 / 2$ Red Angus.

A condição corporal dos animais foi avaliada segundo escala de 1 a 5 (Houghton et al., 1990), adaptada para intervalos de 0,25 . A CC de todos os animais do experimento foi avaliada mensalmente, a partir do diagnóstico de gestação em junho de 2004 até fevereiro de 2005. Todas as avaliações foram feitas por dois técnicos (sempre os mesmos), sendo utilizada na análise estatística a média da avaliação dos dois técnicos. Durante todo o período experimental, os animais foram mantidos sempre juntos, em módulo com três piquetes de Brachiaria decumbens, com pastejo rotacionado entre eles. 
O experimento II, delineado para avaliar o efeito de CC e DPP na resposta ao protocolo de IATF, que usa dispositivo intravaginal de progesterona associado ao estradiol (Fig. 1), descrito por Meneghetti et al. (2005b), foi realizado em duas propriedades de gado de corte comercial:
Fazenda Ponte Pedra (Faz. 1), localizada no município de Tori Xoréu, região do Vale do Araguaia no estado do Mato Grosso, e Fazenda Rio Paraíso (Faz. 2), no município de Jataí, região sudoeste do estado de Goiás.

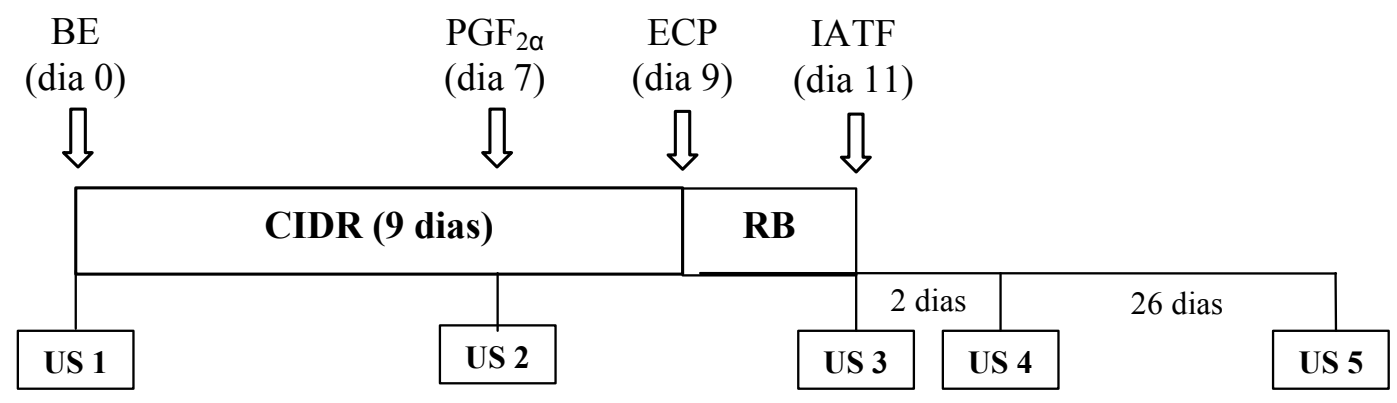

Figura 1. Esquema do protocolo de IATF utilizado no experimento II.

$\mathrm{BE}$ (benzoato de estradiol, Estrogin ${ }^{\circledR}, 2,0 \mathrm{mg}, \mathrm{IM}$ ); $\mathrm{CIDR}^{\circledR}$ (dispositivo intravaginal de progesterona, 1,9g); $\mathrm{PGF}_{2 \alpha}$ (Lutalyse $^{\circledR}, 2,5 \mathrm{ml}, \mathrm{IM}$ ); $\mathrm{ECP}^{\circledR}$ (cipionato de estradiol, 0,5 mg,IM); US: ultra-som; RB: remoção de bezerros; IATF: inseminação artificial em tempo fixo.

Na Faz. 1, foi realizada a IATF em 183 matrizes de dois grupos genéticos que estavam entre 36 e 104 DPP (média de 68 $\pm 19,7$ dias), sendo 60 primíparas da raça Nelore e 123 primíparas 1/2 Nelore $+1 / 2$ Red Angus. Na Faz. 2, usaram-se 355 vacas primíparas da raça Nelore que estavam entre 33 e 97 DPP (média de 59 $\pm 14,6$ dias). As duas propriedades apresentavam manejo nutricional semelhante, com pastejo convencional de Brachiaria decumbens e suplementação apenas de mistura mineral $(9 \%$ de fósforo) fornecida ad libitum.

No experimento II, a CC foi avaliada no momento da IATF, segundo escala de 1 a 5 , adaptada para intervalos de 0,25. A taxa de ciclicidade foi avaliada por meio de dois exames de ultra-som ${ }^{1}$ (com transdutor linear de 5,0 $\mathrm{MHz}$ ), sendo considerados como ciclando os animais que apresentaram tecido lúteo em, pelo menos, um dos dois exames, realizados no dia do início do protocolo (US1, Fig. 1) e sete dias depois (US2, Fig. 1). A taxa de sincronização foi avaliada apenas na Faz. 1, por dois exames de ultra-som (US3 e 4, Fig. 1), e determinada de acordo com a presença e ausência de folículo dominante no momento da IATF e 48 horas depois, respectivamente; também na Faz. 1, foi mensurado o diâmetro do maior folículo presente

${ }^{1}$ Aloka - modelo SSD 500V - Japão. no momento da IATF (US 3, Fig. 1). O diagnóstico de gestação (US5, Fig. 1) foi realizado 28 dias após a IATF.

A IATF foi realizada por técnicos treinados e, no processo de descongelamento do sêmen, foi empregado descongelador eletrônico ${ }^{2}$, para facilitar e padronizar o processo.

No experimento I, a alteração na CC ao longo do período observado foi analisada pelo PROC MIXED do SAS (User's ..., 2003), sendo incluídos na análise os efeitos fixos de grupo genético, mês de parição, mês de avaliação da CC (medida repetida) e respectivas interações, e os efeitos aleatórios de vaca dentro de cada combinação de grupo genético com mês de parição e residual. Admitiu-se para as medidas repetidas um efeito de auto-regressão de primeira ordem. A comparação entre as médias de CC em dezembro (mês em que os animais dos dois grupos genéticos apresentaram a menor CC), observadas para cada combinação de grupo genético com mês de parição, foi feita pelo teste Bonferroni, com nível de significância 0,0083 $(0,05 / 6)$.

No experimento II, a análise estatística das variáveis binárias foi realizada pelo PROC

${ }^{2}$ Fertilize Ltda - Brasil. 
LOGISTIC do SAS (User's ..., 2003). Na análise da taxa de sincronização, foram considerados, no modelo, os efeitos de grupo genético e CC; ciclicidade, DPP e possíveis interações não foram mantidas por não mostrarem efeito significativo $(\mathrm{P}>0,10)$. Na análise da taxa de concepção, foram considerados, no modelo, os efeitos de grupo genético dentro de fazenda e CC; ciclicidade, DPP, touro, inseminador, e possíveis interações não foram mantidas por não mostrarem efeito significativo $(\mathrm{P}>0,10)$. $\mathrm{Na}$ análise da taxa de concepção das vacas efetivamente sincronizadas, foi mantida apenas a variável grupo genético; CC, ciclicidade, DPP, touro, inseminador e possíveis interações não foram mantidas por não mostrarem efeito significativo $(\mathrm{P}>0,10)$. Os efeitos dos DPP na $\mathrm{CC}$ no momento da IATF e o da CC no diâmetro do folículo ovulatório foram analisados por regressão linear por meio do PROC GLM do SAS (User's..., 2003).

\section{RESULTADOS}

No experimento I, foi observado efeito do mês de parição $(\mathrm{P}<0,001)$, mês de avaliação da $\mathrm{CC}$ $(\mathrm{P}<0,0001)$ e interação mês de parição $\mathrm{x}$ mês de avaliação $(\mathrm{P}<0,0001)$ na dinâmica da $\mathrm{CC}$ ao longo do experimento. Os animais com parto no início da estação de parição apresentaram redução mais acentuada na CC (Fig. 2). Também foi evidenciada interação $(\mathrm{P}<0,01)$ grupo genético $\mathrm{x}$ mês de parição, de forma que as vacas Nelore paridas em novembro tiveram maior redução na $\mathrm{CC}$ do que as $1 / 2$ Nelore + 1/2 Red Angus (Fig. 2).

No mês de dezembro, os animais dos quatro grupos de parição nos dois grupos genéticos atingiram a menor CC (Fig. 2). Neste momento, os animais $1 / 2$ Nelore $+1 / 2$ Red Angus paridos em setembro e outubro apresentaram CC semelhante, porém menor $(\mathrm{P}<0,05)$ que os animais paridos em novembro e dezembro (Tab. 1). A redução pós-parto na $\mathrm{CC}$ das vacas cruzadas em relação ao mês de dezembro foi $0,92,0,45$ e 0,16 (pontos na escala 1 a 5 ) para os animais com parto em setembro, outubro e novembro, respectivamente (Tab. 1). Nos animais da raça Nelore, a CC em dezembro dos animais paridos em setembro foi menor $(\mathrm{P}<0,05)$ que o das vacas paridas em novembro e dezembro; já a $\mathrm{CC}$ das paridas em outubro foi menor $(\mathrm{P}<0,05)$ apenas ao das vacas de dezembro. A redução na $C C$ pósparto das vacas Nelore foi $0,91,0,54$ e 0,13 para os animais com parto nos meses de setembro, outubro e novembro, respectivamente (Tab. 1).
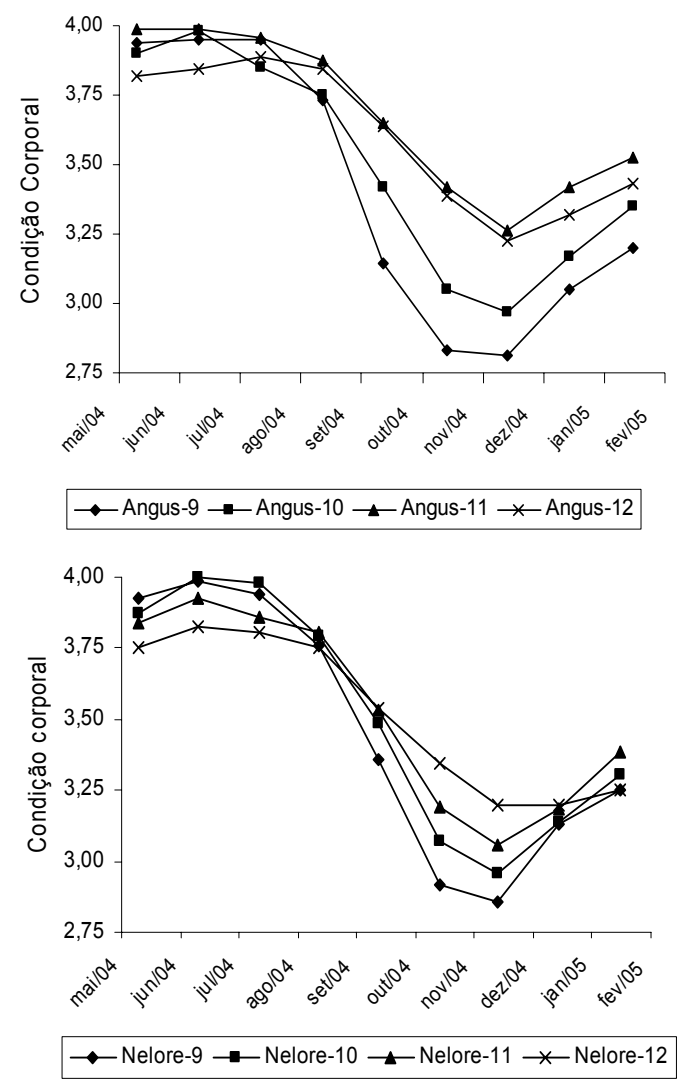

Figura 2. Condição corporal (médias dos quadrados mínimos) no pré e pós-parto de vacas primíparas Nelore e $1 / 2$ Nelore + $1 / 2$ Red Angus, de acordo com o mês de parição.

No experimento II, foi observado maior CC nos animais com menor DPP no momento da IATF. Também foi observada interação DPP versus fazenda versus grupo genético na condição corporal $(\mathrm{P}<0,0001)$. Nas vacas $1 / 2$ Nelore $+1 / 2$ Red Angus (Faz. 1) ocorreu menor redução na $\mathrm{CC}$ do que nas vacas Nelore nas duas fazendas (Fig. 3).

No início do protocolo de IATF, 5,4\% (29/538) dos animais estavam ciclando e não se observou efeito de ciclicidade sobre as taxas de sincronização e concepção.

Foi observado efeito $(\mathrm{P}<0,05)$ da $\mathrm{CC}$ na taxa de sincronização, sendo que os animais com menor $\mathrm{CC}$ tiveram menor sincronização (Fig. 4) e a taxa de sincronização média foi de $91,8 \%$ (168/183). As vacas com menor CC também apresentaram folículos de menor diâmetro no momento da IATF $(\mathrm{P}<0,05$; Fig. 4). Também foi verificado efeito $(\mathrm{P}<0,001)$ de grupo genético no diâmetro folicular, sendo de $11,8 \pm 0,28 \mathrm{~mm}$ nas vacas Nelore $\mathrm{e}$ $13,0 \pm 0,19 \mathrm{~mm}$ nas vacas $1 / 2$ Nelore $+1 / 2$ Red Angus. 
Tabela 1. Condição corporal em dezembro e redução da condição corporal $(\mathrm{CC})$ pós-parto de acordo com o mês de parição em animais de primeira cria da raça Nelore e nas vacas $1 / 2$ Nelore $+1 / 2$ Red Angus

\begin{tabular}{lcccc}
\multicolumn{1}{c}{ Raça } & Mês do parto & $\mathrm{N}$ & $\begin{array}{c}\text { CC* em } \\
\text { dezembro }\end{array}$ & $\begin{array}{c}\text { Redução da CC pós-parto** } \\
\text { (pontos, escala 1 -5) }\end{array}$ \\
\hline 1/2 Red Angus & setembro & 24 & $2,81^{\mathrm{a}}$ & 0,92 \\
$1 / 2$ Red Angus & outubro & 15 & $2,97^{\mathrm{a}}$ & 0,45 \\
$1 / 2$ Red Angus & novembro & 18 & $3,26^{\mathrm{b}}$ & 0,16 \\
& & & & \\
Nelore & setembro & 21 & $2,85^{\mathrm{a}}$ & 0,91 \\
Nelore & outubro & 22 & $2,95^{\mathrm{ab}}$ & 0,54 \\
Nelore & novembro & 29 & $3,06^{\mathrm{bc}}$ & 0,13 \\
Nelore & dezembro & 15 & $3,20^{\mathrm{c}}$ & ----- \\
\hline
\end{tabular}

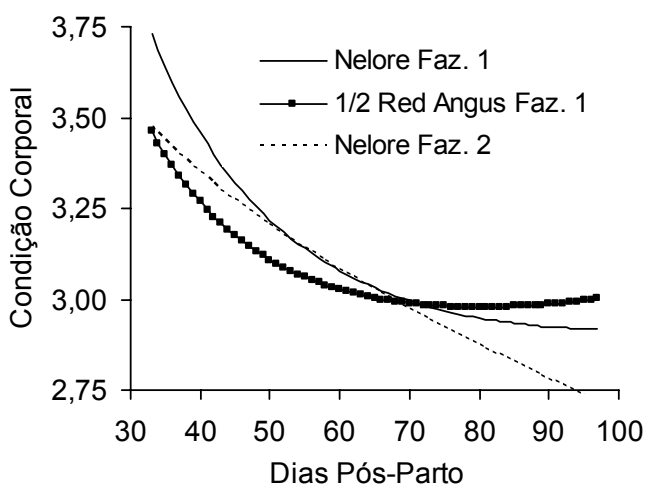

Figura 3. Condição corporal das vacas Nelore e $1 / 2$ Nelore $+1 / 2$ Red Angus na Faz. 1 e vacas Nelore na Faz. 2, de acordo com o número de dias pós-parto.

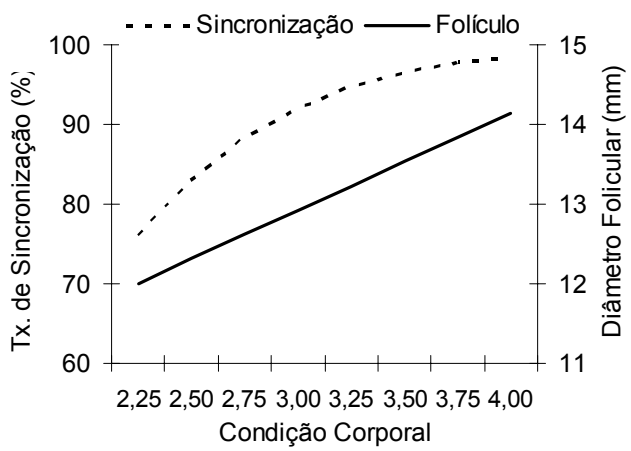

Figura 4. Taxa de sincronização e diâmetro do folículo presente no momento da inseminação artificial em tempo fixo, de acordo com a condição corporal.

Houve efeito da $\mathrm{CC}(\mathrm{P}<0,0001)$ na taxa de concepção. $\mathrm{O}$ aumento na taxa de concepção foi proporcional ao aumento na $\mathrm{CC}$ das matrizes, de forma que o incremento médio na concepção foi de 6 pontos percentuais para cada 0,25 ponto na CC (Fig. 5). A taxa de concepção média foi de
$49,4 \%(266 / 538)$, sendo de $54,1 \%(99 / 183)$ e $47,0 \% \quad(167 / 355)$ nas fazendas I e II, respectivamente. A taxa de concepção das vacas efetivamente sincronizadas foi $58,9 \%(99 / 168)$ e não foi influenciada por nenhuma das variáveis analisadas no estudo (Fig. 5).

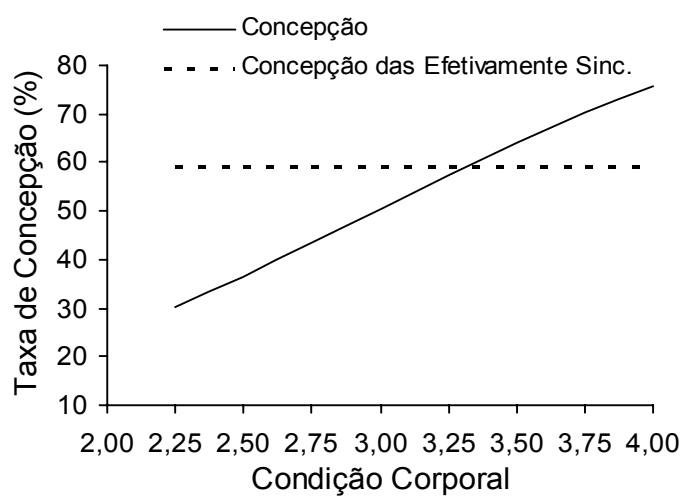

Figura 5. Taxas de concepção e concepção das efetivamente sincronizadas (ovuladas), de acordo com a condição corporal no momento da inseminação artificial em tempo fixo.

\section{DISCUSSÃO}

No experimento I, foi observado que os animais apresentaram menor $\mathrm{CC}$ no mês de dezembro e neste momento as vacas com maior número de dias pós-parto estavam com $\mathrm{CC}$ menor do que as vacas paridas mais recentemente. Na maior parte dos sistemas de estação de monta usados no Brasil, as fêmeas bovinas passam pela fase de maior demanda fisiológica (final de gestação e início de lactação) durante o final das secas. De acordo com o NRC (Nutrient ..., 2000), do oitavo para o nono mês de gestação, ocorre aumento de $55 \%$ na exigência energética para gestação e, do primeiro para o terceiro mês pós-parto, a energia necessária para lactação aumenta $40 \%$ e, se essa 
maior demanda por nutrientes não for atendida pela forragem disponível, resultará em balanço energético negativo, com intensidade variada conforme a qualidade e a quantidade da forragem. Estudos realizados em pastagens de Brachiaria decumbens mostraram redução na disponibilidade de matéria seca, proteína e NDT durante o período de junho a setembro (Euclides e Queiroz, 2000) e menor ganho de peso no mês de setembro (Santos et al., 2004). Esses dados em conjunto mostram que pastagens de Brachiaria decumbens, em setembro, apresentam-se com menor disponibilidade, consumo e qualidade, ocorrendo menor desempenho animal neste momento.

$\mathrm{Na}$ avaliação de dezembro, os animais do experimento I apresentaram a CC mais baixa (Fig. 2), sugerindo que, a partir desse momento, a disponibilidade da forragem começa a atender as necessidades dos animais, possibilitando que estes revertessem o balanço energético negativo pós-parto. Portanto, os animais com parto no final da estação de parição passaram por menor período de restrição alimentar pós-parto e, conseqüentemente, menor redução na CC (Tab. 1). Esses animais que pariram mais tarde na estação de parição tinham no momento da IATF menor número de dias pós-parto e melhor condição corporal, aspectos já observados por Alderton et al. (2000) e Vilela (2004).

Foi verificado acréscimo na taxa de sincronização com o aumento da CC (Fig. 4), independente dos DPP. Protocolos à base de progesterona + estradiol visam à redução nas concentrações de FSH e LH (Burke et al., 2005) e conseqüente atresia folicular (Rhodes et al., 2002) com início de nova onda folicular três a cinco dias após o tratamento (Vasconcelos et al., 1994; Bó et al., 1995), dependendo da dose (Burke et al., 2003) e do éster de estradiol utilizado (Martinez et al., 2005), e independentemente da fase de desenvolvimento em que a onda folicular se encontra (Burke et al., 2001). O grau de balanço energético negativo (BEN) em que o animal se encontra é um fator que pode influenciar $o$ intervalo entre $o$ tratamento com progesterona + estradiol e o início da nova onda folicular. Imakawa et al. (1987) constataram que, em novilhas ovariectomizadas que apresentavam perdas de peso e de CC, o eixo hipotálamo-hipofise tornase hipersensível ao feed back negativo do estradiol sobre a secreção de GnRH, o que influencia negativamente a secreção de LH e FSH (Wiltbank et al., 2002). Esses autores sugeriram que, em vacas em BEN pós-parto, o estradiol pode atuar como na pré-puberdade, inibindo a secreção de GnRH no hipotálamo (Schillo, 1992). Neste experimento, utilizou-se para todas as vacas a dose de $2,0 \mathrm{mg}$ de benzoato de estradiol, dose que em vacas cíclicas provoca inicio de nova onda aproximadamente quatro dias após o tratamento (Burke et al., 2003). Essa dose, entretanto, pode ter tido maior efeito nas vacas com menor $\mathrm{CC}$ devido à maior sensibilidade do hipotálamo ao estradiol, o que poderia atrasar o início da nova onda. Rhodes et al. (2002) observaram que parte das vacas em anestro apresentou atraso na emergência da onda folicular após tratamento com progesterona + estradiol e relacionaram esse efeito com a sensibilidade ao estradiol e à intensidade do anestro. Assim, em animais em anestro com menor CC, o estradiol aplicado no início do protocolo pode ter tido maior capacidade de causar feed-back negativo sobre a atividade secretória dos hormônios no eixo hipotálamohipofise, atrasando o início da nova onda folicular. Dessa forma, o folículo presente no momento da IATF pode ser mais jovem e de menor diâmetro. Neste estudo, nos animais com menor CC e que apresentaram menor sincronização, também verificaram-se folículos menores (Fig. 4), resultado semelhante aos dos estudos de Sá Filho (2006) e Gimenes et al. (2007), em que foram observados menores respostas ao estímulo ovulatório $(\mathrm{GnRH}) \mathrm{em}$ vacas com folículos menores.

A taxa de concepção à IATF foi menor nos animais com menor CC (Fig. 5), semelhante aos resultados obtidos por Cutaia e Bó (2004) e Meneghetti et al. (2005b). Essa menor taxa de concepção das vacas com menor CC foi devido à menor taxa de sincronização ao protocolo. Esse efeito pode ser confirmado ao se analisar a taxa de concepção das vacas efetivamente sincronizadas (Fig. 5), quando a CC não influenciou a concepção. Este resultado, observado nas vacas efetivamente sincronizadas, assemelha-se ao de Souza (2005) que verificou, em vacas leiteiras de alta produção, aumento na taxa de concepção das vacas com menor $\mathrm{CC}$, quando foi aplicado estradiol ao final do protocolo Ovsynch. Vacas que recebem aplicação de estradiol no final de protocolo de 
IATF apresentam maior manifestação de estro do que as que recebem GnRH (Martinez et al., 2002; Souza, 2005). A aplicação de estradiol também aumentou a expressão de estro em vacas que ovularam FD menores (Souza, 2005). Orihuela et al. (1999) demonstraram que o transporte espermático, em ratos, é maior durante o proestro do que em outras fases do ciclo estral e que o tratamento com estradiol-17 $\beta$ exógeno facilita a migração espermática para dentro do oviduto. Nesse caso, o ECP aplicado na retirada do $\mathrm{CIDR}^{\circledR}$ pode ter tido efeito positivo sobre a motilidade uterina e o transporte espermático através do trato reprodutivo, por aumentar os níveis circulantes de estradiol, que estariam mais baixos nas vacas de menor $\mathrm{CC}$ devido à reduzida capacidade esteroidogênica de seus folículos préovulatórios, explicando o aumento na taxa de concepção das vacas com menor CC. De forma concomitante, o período de proestro com estradiol mais alto pela aplicação do ECP pode ter tido efeito positivo sobre a função e ambiente uterino, importante para o desenvolvimento do embrião e do concepto (Day, 2005; Bridges e Day, 2006).

Embora não seja possível confirmar, pode ter ocorrido efeito benéfico do estradiol utilizado no fim do protocolo sobre o ambiente uterino, pois não foram verificados efeitos de CC e DPP na taxa de concepção nos animais efetivamente sincronizados com o protocolo.

\section{CONCLUSÕES}

Os resultados de IATF não foram beneficiados com o aumento dos DPP no início da EM. Vacas com maior CC tiveram maior concepção à IATF. Vacas com maior DPP apresentaram menor CC, sugerindo que propriedades que utilizam essa técnica não devem antecipar a parição de novilhas, visando à maior condição corporal no início de estação de monta.

\section{REFERÊNCIAS BIBLIOGRÁFICAS}

ALDERTON, B.W.; HIXON, D.L.; HESS, B.W. et al. Effects of supplemental protein type on productivity of primiparous beef cows. J. Anim. Sci., v.78, p.30273035, 2000.

BÓ, G.A.; ADAMS, G.P.; CACCIA, M. et al. Ovarian follicular wave emergence after treatment with progestogen and estradiol in cattle. Anim. Reprod. Sci., v.39, p.193-204, 1995.
BÓ, G.A.; CUTAIA, L.; REIS, L. et al. El uso de tratamientos hormonales para mejorar el desempeño reproductivo en ganado de carne en anestro en climas tropicales. In: SIMPOSIO INTERNACIONAL DE REPRODUCCIÓN BOVINA, 1., 2004, Barquisimeto. Anais... Barquisimeto, 2004. p.125-137.

BRIDGES, G.A.; DAY, M.L. Impacto das concentrações de hormônios esteróides sobre a fertilidade de bovinos. In: NOVOS ENFOQUES NA PRODUCAO E REPRODUCAO DE BOVINOS, 10., 2006, Uberlândia. Anais... Uberlândia, 2006. p.175191.

BURKE, C.R.; MUSSARD, M.L.; GRUM, D.E. et al. Effects of maturity of the potential ovulatory follicle on induction of oestrus and ovulation in cattle with oestradiol benzoate. Anim. Reprod. Sci., v.66, p.161174, 2001.

BURKE, C.R.; MUSSARD, M.L.; GASSER, C.L. et al. Estradiol benzoate delays new follicular wave emergence in a dose-dependent manner after ablation of the dominant ovarian follicle in cattle. Theriogenology, v.60, p.647-658, 2003.

BURKE, C.R.; CÁRDENAS, H.; MUSSARD, M.L. et al. Histological and steroidogenic changes in dominant ovarian follicles during oestradiol-induced atresia in heifers. J. Reprod. Fertil., v.129, p.611-620, 2005.

CUTAIA, L.; BÓ, G.A. Factores que afectan los resultados en programas de inseminación artificial a tiempo fijo en rodeos de cría utilizando dispositivos com progesterona. In: SIMPOSIO INTERNACIONAL DE REPRODUCCIÓN BOVINA, 1., 2004, Barquisimeto. Anais... Barquisimeto, 2004. p.109-123.

DAY, M.L. Efeito de estratégias de sincronização da ovulação no desenvolvimento folicular e na concepção. In: NOVOS ENFOQUES NA PRODUCÃO E REPRODUCAO DE BOVINOS, 9., 2005, Uberlândia. Anais... Uberlândia, 2005. p.217228.

EUCLIDES, V.P.B.; QUEIROZ, H.P. Manejo de pastagens para produção de feno-em-pé. Disponível em:

$<$ http://www.cnpgc.embrapa.br/eventos/2000/12encon tro/apostila.html> Acessado em 14 mai. 2007.

GIMENES, L.U.; CARVALHO, N.A.T.; SA FILHO, M.F. et al. Follicle deviation and ovulatory capacity in Bos indicus heifers. Reprod. Fertil. Dev., v.19, p.242, 2007. (Abstr.).

HOUGHTON, P.L.; LEMENAGER, R.P.; MOSS, G.E. et al. Prediction of postpartum beef cow body composition using weight to height ratio and visual body condition score. J. Anim. Sci., v.68, p.1428-1437, 1990. 
IMAKAWA, K.; DAY, M.L.; ZALESKY, D.D. et al. Effects of $17-\beta$ Estradiol in diets varying in energy on secretion of LH in beef heifers. J. Anim. Sci., v.64, p.805-815, 1987.

LAMB, G.C.; STEVENSON, J.S.; KESLER, D.J. et al. Inclusion of an intravaginal progesterone insert plus $\mathrm{GnRH}$ and prostaglandin $\mathrm{F}_{2 \alpha}$ for ovulation control in postpartum suckled beef cows. J. Anim. Sci., v.79, p.2253-2259, 2001.

MARTINEZ, M.F.; KASTELIC, J.P.; ADAMS, G.P. et al. The use of a progesterone-releasing device (CIDR-B) or melengestrol acetate with GnRH, LH, or estradiol benzoate for fixed-time AI in beef heifers. $J$. Anim. Sci., v.80, p.1746-1751, 2002.

MARTÍNEZ, M.F.; KASTELIC, J.P.; BÓ, G.A. et al. Effects of oestradiol and some of its esters on gonadotrophin release and ovarian follicular dynamics in CIDR-treated beef cattle. Anim. Reprod. Sci., v.86, p.37-52, 2005.

MENEGHETTI, M.; LOSI, T.C.; MARTINS Jr., A.P. et al. Protocolos de sincronização de ovulação a base de progesterona e estradiol, em vacas de corte paridas. 2: Avaliação de diferentes doses de eCG. In: CONGRESSO BRASILEIRO DE REPRODUÇÃO ANIMAL, 16., 2005a, Goiânia. Anais... Goiania, 2005a. p.187. (Resumo).

MENEGHETTI, M.; LOSI, T.C.; MARTINS Jr., A.P. et al. Uso de protocolo de IATF associado a diagnóstico precoce de gestação e ressincronização como estratégia para maximizar o número de vacas gestantes por IA em estação de monta reduzida. Hora Vet., v.147, p.25-27, 2005b.

MOSSMAN, D.H.; HANLY, G.J. A theory of beef production. N. Z. Vet. J., v.25, p.96-100, 1977.

NUTRIENT requirements of beef cattle. 7.ed. Washington: National Academy of Sciences, 2000. 232p.

ORIHUELA, P.A.; ORTIZ, M.E.; CROXATTO, H.B. Sperm migration into and through the oviduct following artificial insemination at different stages of the estrous cycle in the rat. Biol. Reprod., v.60, p.908913, 1999.

RHODES, F.M.; BURKE, C.R.; CLARK, B.A. et al. Effect of treatment with progesterone and oestradiol benzoate on ovarian follicular turnover in postpartum anoestrous cows and cows which have resumed oestrous cycles. Anim. Reprod. Sci., v.69, p.139-150, 2002.
RICHARDS, M.W.; SPITZER, J.C.; WARNER, M.B. Effect of varying levels of postpartum nutrition and body condition at calving on subsequent reproductive performance in beef cattle. J. Anim. Sci., v.62, p.300306, 1986.

RUAS, J.R.M.; BRANDÃO, F.Z.; SILVA FILHO, J.M. et al. Indução do estro no pós-parto em vacas primíparas Holandês-Zebu. Arq. Bras. Med. Vet. Zootec., v.57, p.476-484, 2005.

SÁ FILHO, O.G. Efeitos de tratamentos com progesterona elou estradiol na incidência de regressão prematura do corpo lúteo após a primeira ovulação em vacas Nelore pós-parto. 2006. $120 \mathrm{f}$. Dissertação (Mestrado) - Faculdade de Medicina Veterinária e Zootecnia, Universidade Estadual Paulista, Botucatu, SP.

SANTOS, E.D.G.; PAULINO, M.F.; QUEIROZ, D.S. et al. Avaliação de pastagem diferida de Brachiaria decumbens Staf. 2. Disponibilidade de forragem e desempenho animal durante a seca. Rev. Bras. Zootec., v.33, p.214-224, 2004.

SCHILLO, K.K. Effects of dietary energy on control of luteinizing hormone secretion in cattle and sheep. $J$. Anim. Sci., v.70, p.1271-1282, 1992.

SOUZA, A.H. Studies on increasing circulating estradiol in the Ovsynch protocol in high producing lactating dairy cows. 2005. 107f. Dissertação (Mestrado) - Department of Dairy Science, University of Wisconsin, Madison.

USER'S guide: Statistics, Version 9.01. Cary, NC: SAS Institute, 2003.

VASCONCELOS, J.L.M.; PURSLEY, J.R.; WILTBANK, M.C. Effects of syncromate B combined with GnRH on follicular dynamics and time of ovulation. J. Dairy Sci., v.77, p.174, 1994. (Abst.).

VILELA, E.R. Utilização de dispositivo de liberação intravaginal de progesterona no protocolo de sincronização (GnRH/PGF2 $\alpha / G n R H)$ associado a remoção temporária dos bezerros em vacas Nelore paridas. 2004. 58f. Dissertação (Mestrado) Faculdade de Medicina Veterinária e Zootecnia, Universidade Estadual Paulista, Botucatu, SP.

WILTBANK, J.N. Research needs in beef cattle reproduction. J. Anim. Sci., v.31, p.755, 1970.

WILTBANK, M.C.; GUMEN, A.; SARTORI, R. Phisiologycal classification of anovulatory conditions in cattle. Theriogenolgy, v.57, p.21-53, 2002. 